\title{
Nierenzellkarzinom: sechs Jahre Erfahrungen mit Sorafenib
}

- Mittlerweile liegen sechs Jahre klinische Erfahrung mit dem Tyrosinkinase-Inhibitor (TKI) Sorafenib (Nexavar ${ }^{\circledast}$ ) beim fortgeschrittenen Nierenzellkarzinom (mRCC) an über 6.000 Patienten vor. Damit steigen die Chancen, das mRCC in eine chronische Erkrankung mit längerer Überlebenszeit zu überführen. Sorafenib war 2006 der erste Vertreter unter den TKI, die zur Therapie des mRCC von Patienten, bei denen eine vorherige Interferonalpha- oder Interleukin-2-basierte Therapie versagt hat oder die für solch eine Therapie nicht geeignet sind, zugelassen wurden.„Bis dahin hatten wir kaum Therapiemöglichkeiten zur Verfügung“, sagte Dr. Hubert Kübler, München, im Rahmen des Kongresses der Deutschen Gesellschaft für Urologie (DGU) 2012. Die neuen Therapiemöglichkeiten ha- ben nicht nur die Behandlung wesentlich verändert, sondern auch die Prognose der Patienten deutlich verbessert. Eine aktuelle Studie konnte belegen, dass in der frühen Zeit des Einsatzes der neuen Substanzen von 2006 bis 2008 das Gesamtüberleben von median 10,2 auf 17,7 Monate stieg [Harmenberg U et al., GU ASCO 2012, Abstract \#389].

Die Sequenztherapie beim mRCC ist inzwischen der Standard. Kübler gab aber zu bedenken: „Es gibt nicht eine bestimmte Sequenz, sondern jede individuelle Situation erfordert eine speziell abgestimmte Sequenz für den jeweiligen Patienten“. Dabei sollten Komorbiditäten sowie der Allgemeinzustand berücksichtigt werden.

"Sorafenib besitzt einen deutlichen Stellenwert in der Zweitlinientherapie", sagte Küb- ler. So hat Sorafenib in der Sequenztherapie nach Sunitinib für Patienten, die für eine Zytokin-Therapie nicht geeignet sind, klinische Relevanz. In der AXIS-Studie zeigt Sorafenib beim Gesamtüberleben nach Sunitinib eine vergleichbare Effektivität wie Axitinib (16,5 Monate unter Sorafenib vs. 15,2 Monate unter Axitinib) [Rini, et al. Lancet 2011; 378: 1931-9]. Zudem ist Sorafenib nach Sunitinib im Vergleich mit dem mTORInhibitor Temsirolimus in Bezug auf das Gesamtüberleben überlegen, dies zeigen die ersten Veröffentlichungen aus der INTORSECT Studie (16,6 vs. 12,3 Monate) [Hutson TE et al., ESMO 2012, Abstract \# 918].

Nach Informationen von

Bayer, Leverkusen

\section{Elektrotherapie bei Drang- und Belastungsinkontinenz}

- Zur nicht invasiven Behandlung von Belastungs- und Dranginkontinenz steht mit NEUROTECH VITAL ${ }^{\text {TM }}$ ein neues Elektrotherapiegerät zur Verfügung. Erstmalig erfolgt damit die Elektrostimulation zur Behandlung von Belastungsinkontinenz über eine externe Manschette, eine Sonde ist nicht erforderlich.

NEUROTECH VITAL ${ }^{\text {TM }}$ besteht aus einer Geräteeinheit und zwei textilen Manschetten, die rechts und links am Oberschenkel mit Klettverschlüssen fixiert werden. Die Manschetten verfügen über je vier integrierte, großflächige Elektroden mit multipath ${ }^{\mathrm{TM}}$-Technologie, welche die Beckenbodenmuskulatur effektiv stimulieren und die Kontraktionsfähigkeit damit optimal unterstützen. multipath $^{\mathrm{TM}}$ ist eine neue und patentierte Form der Elektrodenkommunikation, bei der alle verfügbaren Elektroden in ständiger Verbindung zueinander stehen, wodurch sämtliche Impulse in wechselndem Rhythmus von einer Körperseite zur anderen gelangen. Mit multipath ${ }^{\mathrm{TM}}$ wird die Beckenbodenmuskulatur großflächig und effektiv gekräftigt. Bereits nach wenigen Wochen verbessern sich die Symptome. Für die Behandlung von Dranginkontinenz steht eine Schmetterlingselektrode (Stimulation des Sakralnerves) zur Verfügung.

Nach Informationen von

Neurotech, Salem

\section{Schutz vor rektalen Nebenwirkungen bei Bestrahlung der Prostata}

— Bei der Radiotherapie des Prostatakarzinoms ist es wichtig, den Tumor möglichst hoch dosiert zu bestrahlen und dabei die angrenzenden Organe zu schonen. Ein wichtiger Schritt in diese Richtung wurde mit der Entwicklung des innovativen SpaceOAR ${ }^{\mathrm{TM}}$-Hydrogels getan. Mit der Applikation dieses Hydrogels in den Denonvillierschen Raum wird für die Dauer der Strahlentherapie ein Abstand zwischen Prostata und Rektumvorderwand von etwa einem Zentimeter geschaffen. Durch diese Distanz kann eine Reduktion der rektalen V70-Strahlendosis von mehr als 83\% erreicht werden [Susil R et al. Int. J. Radiation Oncology Biol Phys 2010; 76: 1251-8]. Das PEG (Polyethylenglykol)-basierte Hydrogel ist ein Applikationssystem mit zwei Komponenten, die über ein Y-Stück vor der Nadel miteinander vermischt werden und innerhalb von acht Sekunden zu einer gelartigen Masse aushärten. Das Gel wird in den Denonvillierschen Raum gespritzt und verbindet sich dort fest für die Dauer von drei Monaten mit Prostata und Rektumvorderwand. Danach wird es durch hydrolytische Prozesse vom Körper abgebaut; nach sechs Monaten ist es im Organismus nicht mehr nachweisbar

Bei der akuten Toxizität wurden laut Prof. Theodor Klotz, Weiden, bei Einsatz des Hydrogels deutlich niedrigere Werte beobachtet. Klotz geht auch von einer deutlichen Reduktion der chronischen Nebenwirkungen aus, was er mittels einer Langzeitbeobachtung der Patienten belegen will. Der limitierende Faktor zur Dosisfindung in der Prostatabestrahlung war die Toxizität auf die Rektumvorderwand. Nach Worten von Klotz wurde bis zur Einführung von SpaceOAR ${ }^{\mathrm{TM}}$ in Weiden die maximale Dosis auf $76 \mathrm{~Gy}$ begrenzt. Jetzt werde mit einer maximalen Dosis von 80 Gy gearbeitet.

Nach Informationen von

CS Diagnostics, Neuss 